\title{
TOWARDS INERTIAL GRADE VIBRATORY MICROGYROS: A HIGH-Q IN-PLANE SILICON-ON-INSULATOR TUNING FORK DEVICE
}

\author{
Mohammad Zaman, Ajit Sharma, Babak Amini, and Farrokh Ayazi \\ School of Electrical and Computer Engineering, Georgia Institute of Technology \\ Atlanta, GA 30332-0250
}

\begin{abstract}
This paper reports on the design and implementation of an in-plane solid-mass single-crystal silicon tuning fork gyro that has potential to achieve inertial-grade performance. A novel design is devised to achieve high $\mathrm{Q}$ in the drive and sense resonant modes $\left(\mathrm{Q}_{\text {drive }}=84,000\right.$ and $\left.\mathrm{Q}_{\text {sense }}=64,000\right)$ with effective decoupling. The gyroscope was fabricated on $40 \mu \mathrm{m}$ SOI using a two-mask process. The drive and sense resonant modes were matched electronically to within $0.07 \%$ of each other and the measured rate results show a sensitivity of $1.25 \mathrm{mV} / \% / \mathrm{s}$ in a bandwidth of $12 \mathrm{~Hz}$.
\end{abstract}

\section{MOTIVATION}

MEMS vibratory gyros have yet to achieve inertial grade performance, i.e., rate resolution and bias stability better than $0.1^{\circ} / \mathrm{h}$. To achieve this, a vibratory gyroscope must attain very high quality factors $(>30,000)$, large sense capacitances $(>1 \mathrm{pF})$, large mass $(>100 \mu \mathrm{g})$ and large drive amplitude $(>5 \mu \mathrm{m})[\mathbf{1}, \mathbf{2}]$. This calls for innovative designs and radical advances in fabrication technology. In an effort to reach this goal, we have introduced an in-plane, solid-mass silicon tuning fork device that incorporates very high $\mathrm{Q}$, a large mass per unit area, and inplane operation within a single framework - unlike previously reported tuning fork gyroscopes (TFG) $[3,4]$. The operating principle is based upon a standard tuning fork's response to rotation. In this design (see Fig.1) the proof-masses are driven to resonance along the $\mathrm{x}$-axis, and the Coriolis acceleration induced by rotation around the $\mathrm{z}$-axis is sensed along the $y$-axis.
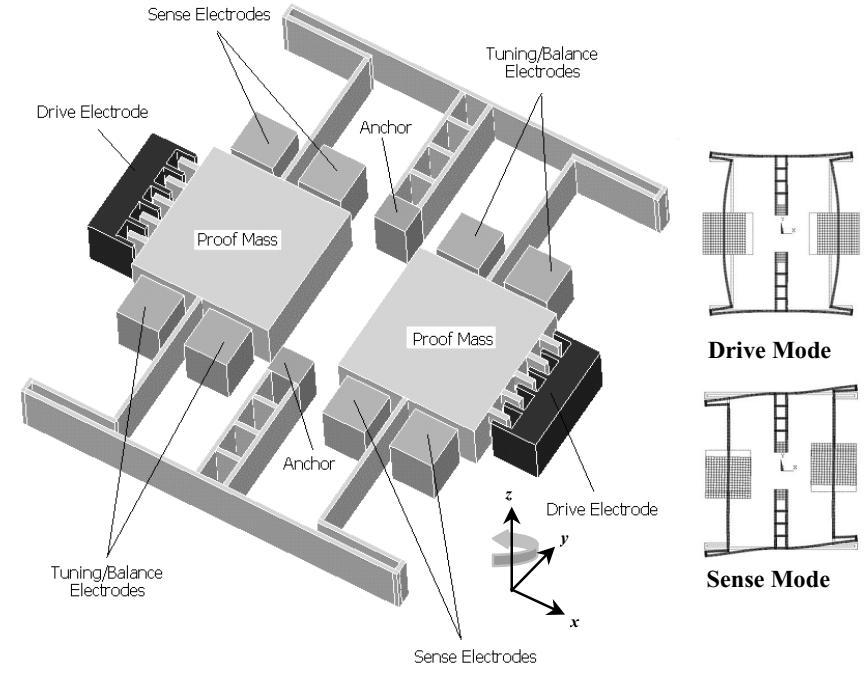

Figure 1. Schematic diagram of the gyroscope and resonant mode shapes as simulated in ANSYS (exaggerated for clarity).

A major incentive in utilizing this symmetric tuning fork architecture as opposed to a frame-design or single-mass-design is the differential sensing capability. As a result, linear acceleration/shock signals are rejected as "common mode" without the need for complex electronics. A high Q in the drive mode is necessary to get large drive amplitudes using small drive voltages. A high $\mathrm{Q}$ in the sense mode is imperative to substantially increase sensitivity of the device and also lower the Brownian noise floor of the gyroscope. The spring structure design makes it possible to drive the solid proof masses linearly with displacement amplitudes in the range of $4-6 \mu \mathrm{m}$. The primary energy loss mechanism that leads to Q degradation is support loss [5]. The anchor and the flexures were carefully designed to ensure minimal support loss and consequently high Q in the drive and sense modes by torque cancellation/reduction. Detailed ANSYS simulations of the flexures were performed to optimize structural dimensions and to allow the sense mode to occur $50-100 \mathrm{~Hz}$ higher than the drive mode. Once the device is fabricated, the sense mode is tuned electrostatically using the tuning electrodes to match the drive mode frequency.

\section{FABRICATION}

The prototype structures were fabricated on $40 \mu \mathrm{m}$ SOI wafers. SEM pictures of a fabricated device are shown in Figure 2.

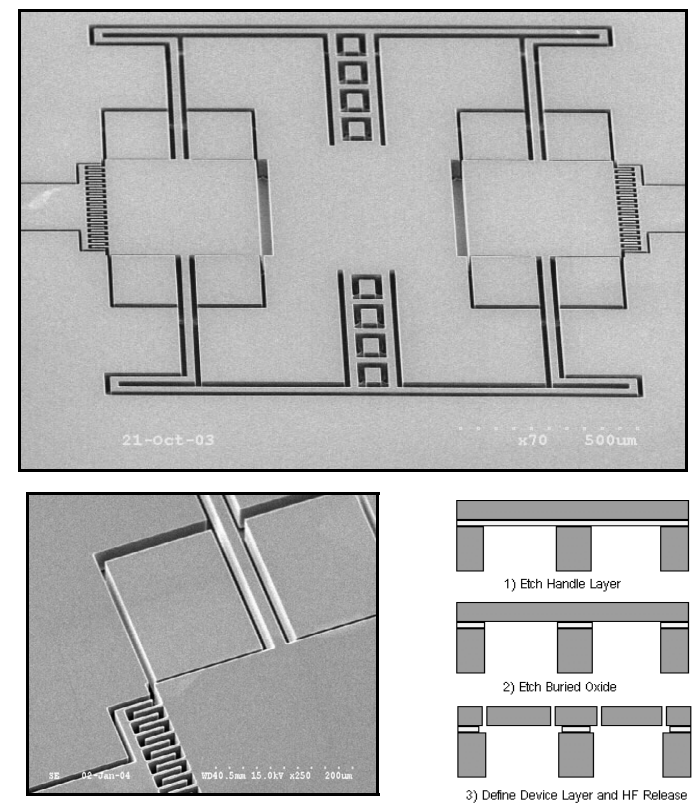

Figure 2. (Clockwise from top) In-Plane Solid-Mass Silicon TFG, process flow, and close-up of $6 \mu \mathrm{m}$ comb \& $2.5 \mu \mathrm{m}$ sense gaps.

The moving sections of the structure and the areas under the comb drives are first released from the backside of the wafer by etching the handle silicon layer through to the buried oxide layer using the Bosch process. The buried oxide is then removed in a reactive ion etching (RIE) system and finally the top layer is patterned all the way through, leaving behind a suspended structure whose anchors are supported to the handle substrate via several support posts. The final etch step involves a quick HF etch to release areas under these supports. Such a fabrication flow is simple and precludes the requirement of any perforations in the proof mass, resulting in a larger mass per unit area. The simultaneous elimination of the ground plane under the comb drives prevents the excitation of the out of plane modes and detrimental effects of levitation. 


\section{EXPERIMENTATION}

A prototype device was tested in vacuum and high $\mathrm{Q}$ operation was confirmed. Figure 3 shows the measured drive and sense resonant modes respectively with high $\mathrm{Q}$ values $(\sim$ 81,000 for drive and $\sim 64,000$ for sense). As discussed earlier, such high Q's are essential to achieve inertial grade performance in a small bandwidth. Larger bandwidth can be obtained by operating the device in closed loop at the expense of sensitivity.
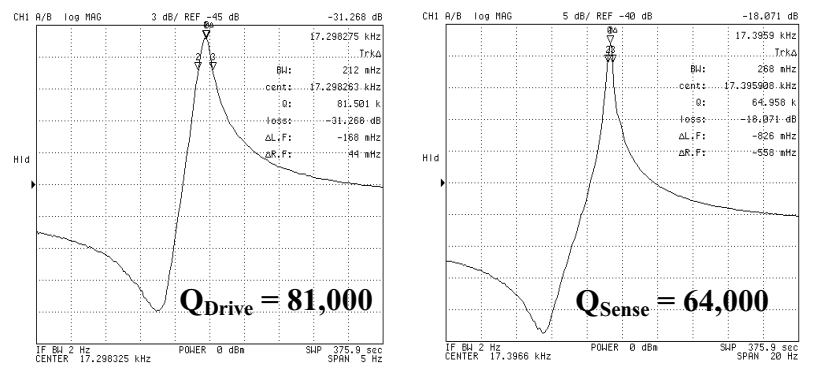

Figure 3. Measured drive and sense resonant modes.

The drive and sense frequencies were matched to within $0.07 \%(12 \mathrm{~Hz}$ over $17.5 \mathrm{kHz})$ of each other (see Figure 4$)$. Despite a mechanically decoupled design, in practice, fabrication imperfections can lead to non-zero off-diagonal elements in the spring stiffness and damping coefficient matrices [6] resulting in significant zero-rate output (ZRO). This quadrature error prevents close matching of the drive and sense resonant modes [4]. Inertial grade performance requires that the high $\mathrm{Q}$ resonant modes be matched. This lowers the Brownian noise floor by a factor of $\sqrt{ } \mathrm{Q}_{\text {sense }}[\mathbf{1}]$ and improves stability. The Brownian noise floor is an order of magnitude greater than the electronic noise floor and is therefore the key factor in determining the resolution of the gyroscope. Additionally, any mismatch is undesirable as it reduces the sensitivity and makes it a function of the frequency of input rotation signal.

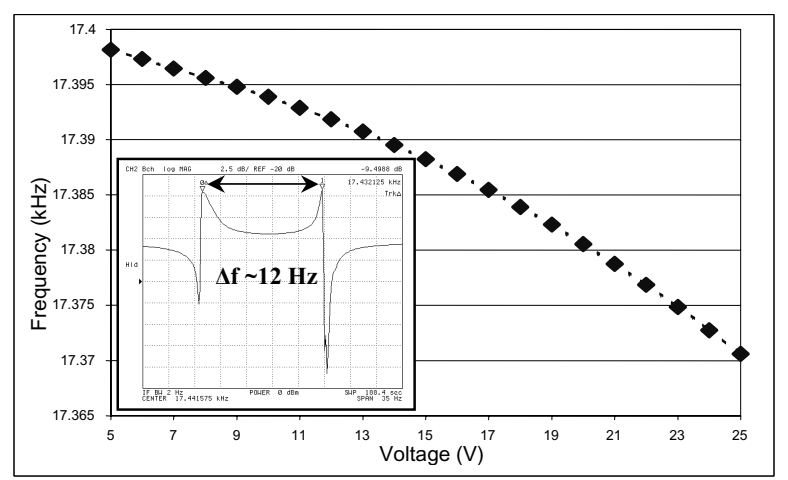

Figure 4. Tuning characteristics of sense mode frequency. Inset: The resonant modes have been matched to within $12 \mathrm{~Hz}$.

Measured rate results from the tuning fork gyro with a $12 \mathrm{~Hz}$ resonant mode mismatch and $1 \mu \mathrm{m}$ drive amplitude yields an open-loop rate sensitivity of $1.25 \mathrm{mV} /{ }^{\circ} \mathrm{s}$, as shown in Figure 5. Table I summarizes the key gyroscope parameters.

\section{CONCLUSIONS AND FUTURE DIRECTIONS}

An in-plane, solid-mass silicon tuning fork gyroscope has been designed and implemented on $40 \mu \mathrm{m}$ SOI. This design demonstrates high drive and sense mode quality factors, large drive amplitudes and increased mass - all critical requirements to lower the Brownian noise floor and thereby achieve subdegree per hour angular rate resolutions.

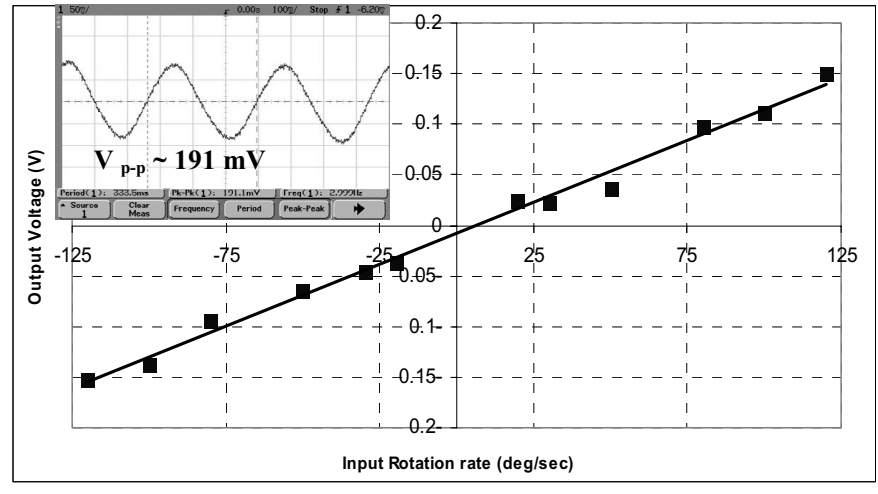

Figure 5. Output voltage versus input rotation rate. Inset: The response of the sensor to an input of $150 \% 3 \mathrm{~Hz}$ signal.

However, the large capacitance requirements are restricted by the aspect ratio limitations of the current process flow. The HARPSS process [2] is being employed to overcome the aspect ratio problem and increase the sense capacitance to $>1 \mathrm{pF}$. Finally, strategic electrode placement schemes and electronic feedback control mechanisms are being investigated to achieve complete matching of the high-Q resonant modes and cancel the quadrature error. The high $Q$ operation calls for advancements in vacuum packaging technologies [7], which is being pursued as well.

Table I: Summary of the solid-mass Silicon TFG parameters.

\begin{tabular}{|l|r|}
\hline Single proof mass dimension (in $\mu \mathrm{m}$ ) & $400 \times 400 \times 40$ \\
\hline Effective mass & $30 \mu \mathrm{g}$ \\
\hline Drive mode resonant frequency (in kHz) & $\begin{array}{r}\text { ANSYS: } 17.413 \\
\text { Measured: } 17.298\end{array}$ \\
\hline Sense mode resonant frequency (in kHz) & $\begin{array}{r}\text { ANSYS: } 17.458 \\
\text { Measured: } 17.396\end{array}$ \\
\hline Split of drive and sense resonant frequencies & $12 \mathrm{~Hz}$ \\
\hline Rate sensitivity & $1.25 \mathrm{mV} / \% / \mathrm{s}$ \\
\hline Resolution with 12 Hz freq mismatch & $0.01^{\circ} / \mathrm{s} / \sqrt{\mathrm{Hz}}$ \\
\hline Theoretical Brownian noise floor with matched modes & $0.3 \% / \mathrm{h} / \sqrt{\mathrm{Hz}}$ \\
\hline Electronic noise floor (input ref. noise $=1 \mu \mathrm{V} / \sqrt{\mathrm{Hz}}$ ) & $0.02^{\circ} / \mathrm{h} / \sqrt{\mathrm{Hz}}$ \\
\hline Measured temp. coefficient of freq. (TCF) & $-59 \mathrm{ppm} /{ }^{\circ} \mathrm{C}$ \\
\hline
\end{tabular}

\section{ACKNOWLEDGEMENT}

This work is supported under the DARPA HERMIT program. The authors wish to thank Dr. Zhili Hao for ANSYS assistance. Travel support has been generously provided by the Transducers Research Foundation and by the DARPA MEMS and BioFlips programs.

\section{REFERENCES}

[1] Ayazi, F., "A High Aspect-Ratio High-Performance Polysilicon Vibrating Ring Gyroscope," Ph.D. Dissertation, University of Michigan, Ann Arbor (2001).

[2] Ayazi, F. and Najafi, K., "A HARPSS Polysilicon Vibrating Ring Gyroscope" IEEE/ASME JMEMS, June 2001, pp. 169-179.

[3] Bernstein, J., et al., "A Micromachined Comb-Drive Tuning Fork rate gyroscope," Proceedings MEMS 1993, pp. 143-148.

[4] Schwarzelbach, O., et al., "New Approach for Resonant Frequency Matching of Tuning Fork Gyroscopes by Using a NonLinear Drive Concept," Proceedings Transducers 2001, pp.464-467. [5] Z. Hao, et al., "An Analytical Model for Support Loss in Micromachined Beam Resonators with In-plane Flexural Vibrations," Sensors and Actuators A, Vol. 109, Dec. 2003, pp.156-164.

[6] Clark. W. A., "Micromachined Vibratory Rate Gyroscopes," Ph.D. Dissertation, University of California, Berkeley (1997).

[7] Najafi, K., "Micropackaging Technologies for Integrated Microsystems: Applications to MEMS and MOEMS," Proc. SPIE's Micromachining \& Microfabrication Symp. 2003, pp.1-19. 